\title{
THE SPATIAL STRUCTURE OF BUBBLE PINCH-OFF*
}

\author{
M. A. FONTELOS ${ }^{\dagger}$, J. H. SNOEIJER ${ }^{\ddagger}$, AND J. EGGERS $\$$
}

\begin{abstract}
We have previously found [J. Eggers, M. A. Fontelos, D. Leppinen, and J. H. Snoeijer, Phys. Rev. Lett., 98 (2007), 094502] that the pinch-off of a gas bubble in an inviscid environment is controlled by scaling exponents which are slowly varying in time. To leading order, these results did not require the spatial profile of the interface near break-up. Here we refine our previous analysis by computing the entire shape of the neck. The neck shape is characterized by similarity functions that are also slowly varying on a logarithmic scale. We compare these results to experiments and find agreement within the experimentally accessible range. More detailed confirmation of the asymptotic analysis is provided by the excellent agreement with numerical simulations of the bubble pinch-off.
\end{abstract}

Key words. capillary breakup, finite-time singularities

AMS subject classifications. 35Q35, 76B45, 35L67

DOI. $10.1137 / 090776470$

1. Introduction. In this paper, we address the collapse of a cavity or the pinchoff of a bubble in a body of fluid whose viscosity is sufficiently small as to be negligible. A typical experimental situation is shown in Figure 1.1: an air bubble is released from a pipette submerged under water. As the bubble rises, it pinches off at a point, preserving radial symmetry [23]. The purpose of the present paper is to find the asymptotic form of the profile around the pinch-point as the minimum neck radius goes to zero. For the rest of this paper, we will assume that the pinch-point is located at the origin $z=0$.

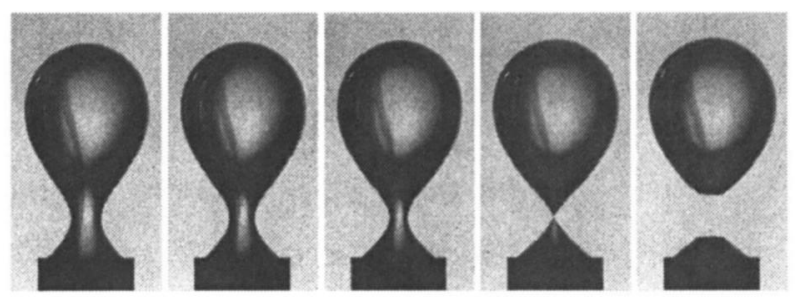

FIG. 1.1. The pinch-off of an air bubble in water. The air is released from a nozzle whose outer radius is $R_{0}=1.57$. The time between two successive panels is $0.5 \mathrm{~ms}$. (Reprinted with permission from Thoroddsen, Etoh, and Takeora, Phys. Fluids, 19 (2007), 042101. Copyright 2007, American Institute of Physics.]

Another important case is that of a cavity [8] that is produced near the surface of water, for example by the impact of a solid object. Recently, there has been a

${ }^{*}$ Received by the editors November 9,2009 ; accepted for publication (in revised form) June 20 , 2011; published electronically September 22, 2011.

http://www.siam.org/journals/siap/71-5/77647.html

†'Instituto de Ciencias Matemáticas (ICMAT, CSIC-UAM-UCM-UC3M), C/ Serrano 123, 28006 Madrid, Spain (marco.fontelos@icmat.es).

${ }^{\ddagger}$ Physics of Fluids Group, University of Twente, P.O. Box 217, 7500 AE Enschede, The Netherlands (j.h.snoeijer@tnw.utwente.nl). This author's work was supported by a Marie Curie European Fellowship FP6 (MEIF-CT2006-025104).

$\S$ School of Mathematics, University of Bristol, University Walk, Bristol BS8 1TW, UK (jens. eggers@bristol.ac.uk). 
flurry of experiments using the bubble $[4,16,18,23]$ or the cavity geometry $[2,8]$ in water. In the presence of perturbations, axisymmetry can be broken significantly $[18,2]$. However, if the bubble is sufficiently small, the shape is perfectly axisymmetric down to the smallest experimentally observed scale of a few $\mu \mathrm{m}$. It was also confirmed experimentally that the air inside the bubble, as well as the viscosity of the surrounding fluid, has a negligible effect on the dynamics [23]. However, even in water, a tiny satellite bubble about $5 \mu \mathrm{m}$ in diameter is observed after break-off occurs. If the viscosity of the surrounding fluid or the density of the gas is increased, the dynamics changes [23]. In particular, the size of satellite bubbles becomes substantial [15].

A naive expectation would have been that the pinch-off of a bubble in water is governed by the same scaling laws as the "inverse" case of a drop of water pinching off in air, for which the minimum drop radius scales like $h_{0} \propto t^{\prime 2 / 3}$, where $t^{\prime}=t_{0}-t$ and $t_{0}$ is the pinch-off time $[6,7]$. This scaling law follows from the simple physical picture of pinch-off being driven by surface tension and resisted by inertia [22]. If $\gamma$ is the surface tension and $\rho$ the density, $\left(\gamma t^{\prime 2} / \rho\right)^{1 / 3}$ is the only local length scale. Surprisingly, the scaling exponent $\alpha$ for bubble pinch-off was found to be close to $1 / 2$, the value proposed by [20] and [21]. This implies faster pinch-off than anticipated, and surface tension must become subdominant, inertia being the only remaining factor.

In [12] it was shown that the scaling behavior of bubble pinch-off is described by a scaling exponent $\alpha(\tau)=1 / 2+1 /(4 \sqrt{\tau})$, where $\tau=-\ln t^{\prime}$. The approach to the asymptotic limit $1 / 2$ is therefore exceedingly slow. When evaluating at experimentally accessible time scales, one indeed obtains values significantly larger than $1 / 2$ and consistent with experimental values of $0.56[18]$ and 0.57 [23] reported in the literature. Note that there is no dimensional argument for the value of $1 / 2$ : as surface tension drops out of the leading order balance, the equation of motion becomes invariant under a change in time scale. This also implies that there is no intrinsic time scale by which to make $t^{\prime}$ dimensionless, and thus $\tau$ is defined only up to a constant shift $\tau_{0}$. The value of $\tau_{0}$ is nonuniversal and set by the initial condition.

The results of [12] were obtained by considering local quantities near the pinchpoint alone, namely the minimum radius and the curvature. In the present paper we reveal the surprising spatial profile of the neck. We show that the pinch region is characterized by similarity profiles, which themselves are logarithmically dependent on time. We find that the profile is symmetric around the pinch-point, while away from the neck the radius increases as a power law with an exponent that is once more slowly varying in time. Both features are consistent with experimental observations and are verified in more detail using numerical simulations. As an added benefit of our analysis, we obtain subleading expressions for the time dependence of the exponent for the neck radius.

\section{Description of the dynamics.}

2.1. Source distribution. We would like to solve the inviscid, irrotational, axisymmetric flow problem outside a cavity, which we model as being at constant pressure. The axis of symmetry is aligned with the direction of gravity, as shown, for example, in the experimental images of Figure 1.1. The velocity $\mathbf{u}$ can thus be written as

$$
\mathbf{u}=\nabla \Phi, \quad \Delta \Phi=0
$$


Our approach is based on representing the velocity potential by point sources distributed along the centerline of the cavity of length $2 L[1]$ :

$$
\Phi=\int_{-L}^{L} \frac{C(\xi, t) d \xi}{\sqrt{(z-\xi)^{2}+r^{2}}} .
$$

It remains to be seen whether a given flow can be represented in the form (2.2). However, we stress that $(2.2)$ is an exact solution of (2.1), with vanishing flow at infinity. When used in slender-body theory, the kernel of the integral in (2.2) is usually expanded in a parameter $\epsilon$ characterizing the slenderness, while we avoid such an expansion for the moment.

We assume that there are no overhangs, so the shape of the cavity can be represented by the local radius $h(z, t)$. As usual, the motion of the surface is determined by the kinematic boundary condition

$$
\partial_{t} h+h^{\prime} u_{z}=\left.u_{r}\right|_{r=h(z, t)},
$$

where the prime denotes the derivative with respect to the spatial argument. Using $(2.2)$, the velocity on the surface is given by

$$
\begin{aligned}
& u_{z}(z, t)=-\int_{-L}^{L} \frac{C(\xi, t)(z-\xi) d \xi}{{\sqrt{(z-\xi)^{2}+h^{2}(z, t)}}^{3}}, \\
& u_{r}(z, t)=-\int_{-L}^{L} \frac{C(\xi, t) h(z, t) d \xi}{{\sqrt{(z-\xi)^{2}+h^{2}(z, t)}}^{3}} .
\end{aligned}
$$

Integrating Euler's equation from infinity (where the pressure is assumed to vanish) to the surface, one obtains

$$
\partial_{t} \Phi+(\nabla \Phi)^{2} / 2=-p /\left.\rho\right|_{r=h(z, t)}
$$

where $p$ is the pressure on the exterior of the surface. Thus if $p_{0}$ is the pressure in the cavity, by Laplace's formula [19] we have $p-p_{0}=-\gamma \kappa+\rho g Z$, where $\kappa$ is (twice) the mean curvature, $\rho$ is the fluid density, $g$ is the acceleration of gravity, and $Z$ denotes the depth from the fluid surface. Thus we arrive at the following equation for the source distribution $C(z, t)$ :

$$
\int_{-L}^{L} \frac{\partial_{t} C(\xi, t) d \xi}{\sqrt{(z-\xi)^{2}+h^{2}(z, t)}}+\frac{u_{z}^{2}+u_{r}^{2}}{2}=-\frac{p_{0}}{\rho}+\frac{\gamma}{\rho} \kappa+g Z .
$$

Equations (2.3), (2.4), and (2.6) form a closed system of equations for $h(z, t)$ and $C(z, t)$, which can be studied numerically. Although the system comes from the ansatz (2.2), which is usually associated with slender-body theory, this system is formally an exact solution of the full inviscid, irrotational flow problem that we set out to solve. However, a problem with the representation (2.2) is that not all smooth irrotational flows can be represented in that way. For example, if one places a delta function on the axis inside the cavity, this produces a perfectly acceptable flow on the outside of the cavity yet cannot be written in the form (2.2) with a smooth $C$. This means not all initial conditions can be realized by using the formulation (2.3), (2.4), (2.6). In particular, there is a problem in describing the motion of a closed surface, as the following example of a steadily moving spherical bubble shows. 
The main problem in describing the flow field around a closed cavity in the form (2.2) arises near the end of the cavity, where the shape is no longer slender. How this problem can be dealt with in a perturbation expansion [17] has been demonstrated. However, this issue is not relevant to the dynamics of pinching, which is a localized phenomenon, independent of flow conditions away from the pinch-point. We therefore avoid the description of a closed bubble, but rather allow for a finite bubble radius at the boundaries of the computational domain. Under these conditions our simulations of $(2.3),(2.4),(2.6)$ indicate that $C(z, t)$ remains smooth at all times before the real pinch-off singularity occurs. As far as we are aware, this system has not been derived or studied before.

2.2. Slender body. To make analytical progress, we wish to derive an approximation of $(2.3),(2.4),(2.6)$ that is valid for slender cavities. We define $h_{0}$ as the minimum radius of the cavity, which we take to occur at $z=0$, and $\Delta$ measures the width of the pinch region:

$$
h_{0}=h(0, t), \quad \Delta=\left(\frac{2 h_{0}^{2}}{\left(\partial_{z}^{2} h^{2}\right)(0, t)}\right)^{1 / 2} .
$$

Throughout, we will be making the approximation that $h_{0}$ is much smaller than $\Delta$. This suggests the introduction of the small aspect ratio parameter

$$
\epsilon(\tau)=h_{0} / \Delta
$$

We will now perform a systematic expansion in $\epsilon$ to derive a simplified integral equation, previously introduced by us [12] on the basis of intuitive arguments. Corrections to the leading slender-body asymptotics will be smaller by a factor of the order of the aspect ratio $\epsilon(\tau)$, as expected. We will see below that $h_{0}(\tau) \approx e^{-\tau-\sqrt{\tau}}$ and $\epsilon(\tau) \approx e^{-\sqrt{\tau} / 2}$, so the aspect ratio is indeed small in the limit of $\tau=-\ln t^{\prime} \rightarrow \infty$. In particular, $u_{z}=\partial_{z} \Phi \approx \epsilon(\tau) \partial_{r} \Phi \ll \partial_{r} \Phi=u_{r}$, so only the radial part of the velocity field needs to be considered in (2.6).

The integral for $u_{r}$ (cf. (2.4)) is dominated by local contributions, as one finds by considering the substitution $\eta=(z-\xi) / h(z)$, which gives

$$
u_{r}(z)=-\frac{1}{h(z)} \int_{\frac{z+L}{h(z)}}^{\frac{z-L}{h(z)}} \frac{C(z-\eta h(z))}{\left(1+\eta^{2}\right)^{\frac{3}{2}}} d \eta
$$

To find the leading order contribution as well as corrections, we expand $C$ according to Taylor's theorem:

$$
C(z-\eta h(z))=C(z)-C^{\prime}(z) \eta h(z)+C^{\prime \prime}\left(z_{0}\right) \eta^{2} h^{2}(z) / 2,
$$

where $z_{0}=z-\eta_{0} h(z)$ for some $\eta_{0}$ in $(-\eta, \eta)$. Since $\Delta$ is the width of the profile, it is safe to assume that $C^{\prime \prime} / C$ is of order $1 / \Delta^{2}$ and $C^{\prime \prime} / C^{\prime}$ is of order $1 / \Delta$ uniformly.

We now introduce (2.10) into (2.9) and estimate the integrals corresponding to each term in (2.10). Hence we have to evaluate

$$
\int_{\frac{z+L}{h}}^{\frac{z-L}{h}} \frac{\eta}{\left(1+\eta^{2}\right)^{\frac{3}{2}}} d \eta \approx \int_{\frac{z+L}{h}}^{\infty} \frac{d \eta}{\eta^{2}}-\int_{-\infty}^{\frac{z-L}{h}} \frac{d \eta}{\eta^{2}}=\frac{h z}{z^{2}-L^{2}} \approx-\frac{h z}{L^{2}}
$$

and

$$
\int_{\frac{z+L}{h}}^{\frac{z-L}{h}} \frac{\eta^{2}}{\left(1+\eta^{2}\right)^{\frac{3}{2}}} d \eta \sim 2 \ln (h / L)
$$


and therefore

$$
u_{r}(z) \approx \frac{-2 C(z)}{h(z)}-C^{\prime}(z) \frac{h(z) z}{L^{2}}+C^{\prime \prime}\left(z_{0}\right) h(z) \ln \left(\frac{h(z)}{L}\right) .
$$

The second term on the right-hand side of (2.11) is much smaller than the third term,

$$
\frac{2 C^{\prime}(z) h z / L^{2}}{C^{\prime \prime}\left(z_{0}\right) h \ln h} \approx \frac{2 \Delta / L}{\ln h}=\Delta O\left(\frac{1}{\ln h}\right) \ll O\left(\frac{1}{\ln \epsilon}\right),
$$

if $|z| \leq L$. The third term, on the other hand, is smaller than the leading term by a factor of

$$
\frac{C^{\prime \prime} h \ln h}{2 C / h} \approx \frac{1}{2} \frac{h_{0}^{2} \ln h_{0}}{\Delta^{2}}=\frac{1}{2} \epsilon^{2} \ln h_{0}=o(\epsilon),
$$

where we have used $\left|\epsilon \ln h_{0}\right| \approx \tau e^{-\sqrt{\tau} / 2} \ll 1$. Hence we conclude

$$
u_{r}(z)=-\frac{2 C(z)}{h(z)}(1+o(\epsilon))
$$

The quality of this leading order approximation,

$$
u_{r}(z) \approx-2 C(z) / h(z),
$$

is tested in Figure 2.2 below. For the analysis to follow, it is convenient to use the cross-sectional area $\pi a(z, t)$ of the cavity, rather than the radius, to describe the slender approximation:

$$
a(z, t) \equiv h^{2}(z, t) .
$$

Accordingly, we set $a_{0}=h_{0}^{2}$. Inserting (2.13) into (2.3) and neglecting $u_{z}$, one obtains $\dot{a}=-4 C$, where a dot denotes the time derivative. Finally, once more neglecting $u_{z}$ relative to $u_{r}$, we arrive at

$$
\int_{-L}^{L} \frac{\ddot{a}(\xi, t) d \xi}{\sqrt{(z-\xi)^{2}+a(z, t)}}=\frac{\dot{a}^{2}}{2 a}-\frac{4 \gamma}{\rho} \kappa+4 g Z+\frac{4 p_{0}}{\rho},
$$

which is accurate up to corrections of order $\epsilon$.

As for the study of the pinch-off singularity, two observations are in order [12]. First, the contribution to the integral is local, so we can effectively set $L \rightarrow \infty$. This results from the spatially localized form of the acceleration $\ddot{a}$, as discussed in section 3 and calculated in section 4 . Second, surface tension as well as gravity is irrelevant to the asymptotic problem. Namely, the asymptotic behavior of the cross-sectional area is $a_{0} \propto t^{\prime}$. This means that the first term on the right-hand side of (2.15) diverges like $t^{\prime-1}$, while the surface tension contribution diverges only like $\kappa \sim h_{0}^{-1} \sim t^{\prime-1 / 2}$, and gravity as well as the cavity pressure remain of order one. Thus the last three terms from the right-hand side of (2.15) can be dropped as far as the asymptotic behavior is concerned. As a result, for most of the paper we will be dealing with the simplified equation

$$
\int_{-\infty}^{\infty} \frac{\ddot{a}(\xi, t) d \xi}{\sqrt{(z-\xi)^{2}+a(z, t)}}=\frac{\dot{a}^{2}}{2 a} .
$$

As we will see below, (2.16) performs very well in describing real experimental data close to pinch-off. We reiterate that (2.16) remains invariant if time is multiplied by any constant. For all our numerical tests, we will approximate the integral on the left by the integral between $-L$ and $L$, as in (2.15). 
2.3. Numerical simulation. We solve (2.3), (2.4), (2.6) using a fully implicit method, using a numerical scheme developed originally by [11]. The solution is described in terms of the two fields $h$ and $C$. In the case of (2.16), the formulation is in terms of $a$ and $\dot{a}$. The time stepping is of second order, and the step size is controlled by comparing a step of size $\Delta t$ with two steps of size $\Delta t / 2$. Adaptive spatial refinement is of course crucial and is performed whenever the minimum $h_{0}$ has changed a certain small percentage. The grid spacing around the pinch-point is based on the width of the profile as determined from the previous solution. After a new grid is found, the old solution is interpolated to the nodes of the new grid. The integrals are evaluated simply over the computational domain, and no other boundary conditions are imposed. Since the phenomenon we describe is local, the precise form of the boundary conditions is not expected to have significant impact on the results.

As long as surface tension is finite, our method works well up to a minimum radius of $h_{0} \approx 10^{-4}$ at least. However, eventually instabilities become more and more difficult to control; this is to be expected, since surface tension drops out from the leading order balance close to the pinch-point and is thus less and less effective at regularizing any short-wavelength instabilities. We therefore opt for a stronger form of regularization, which remains effective for very small $h_{0}$. To this end, we add a correction to the equation, which is small on the scale of the expected pinching solution but which is of higher order in the derivative. This is achieved by adding a term $\epsilon_{\text {reg }} \dot{C}^{\prime \prime} h^{2}$ to the left-hand side of (2.6). Clearly, this term is going to dominate over the integral in the limit of large wave numbers, and thus fixes the ill-posedness. The second derivative is multiplied by $h^{2}$, to make sure it stays of roughly constant size relative to $u_{r}^{2}$, which is driving the motion toward pinch-off.

Namely, $u_{r}$ scales like $C / h$ according to (2.11). In the simplest approximation [12], $C \approx \dot{a} \sim t^{\prime 0}$, and $h^{2} \sim t^{\prime}$, which means that $u_{r}^{2} \sim t^{\prime-1}$. On the other hand, the width of the profile scales like $\Delta \sim t^{\prime 1 / 2}$, and one obtains $\dot{C}^{\prime \prime} / h^{2} \sim t^{\prime-1}$ as well. The prefactor $\epsilon_{\text {reg }}$, which we typically choose to be $10^{-3}$, ensures that the stabilizing term is very small. This is confirmed directly from the simulation and holds true uniformly in space and time. We also checked that a variation of $\epsilon_{\text {reg }}$ by a factor of $10 \mathrm{did}$ not affect the results significantly.

A typical numerical result of the full system of equations (2.3), (2.4), (2.6) is shown in Figure 2.1. The half-width of the domain was $L=4$. The initial condition was chosen symmetric about the origin, and we neglected the effects of surface tension and gravity. In the simulations reported here, the initial cavity radius $R_{0}$ was chosen as the length scale. Since pinch-off is provoked by an inward pointing initial velocity field of maximum value $V_{0} \approx 0.91$, a typical time scale of the simulation is $T=R_{0} / V_{0}$. However, we have not normalized time by this value, since all quantitative comparisons are made allowing for an arbitrary scale factor in the time scale.

For the rest of this paper, we will study the profile in the neighborhood of the point at which the radius goes to zero; see Figure 2.1. This permits us to use the slender-body description (2.16) as a starting point, both for our analytical arguments and for numerical tests. To confirm the quality of approximation of the latter, we took the radial velocity $u_{r}$ as used in the simulation of Figure 2.1, and compared it to its counterpart in the slender-body approximation. In Figure 2.2 we show this comparison for a profile very close to pinch-off. To be able to show more of the profile, we use a logarithmic scale. As expected, the agreement is extremely good and is lost only if the distance from $z=0$ becomes of order one.

We also compared a simulation of the asymptotic equation (2.16) directly to experiment. We chose $L=2$, and once more used a symmetric initial condition, while 


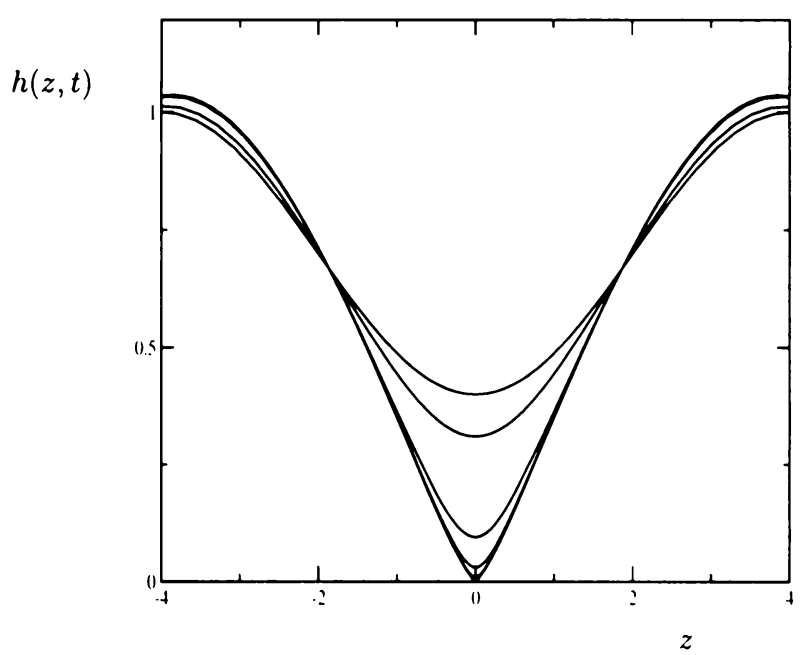

FIG. 2.1. A sequence of profiles leading to pinch-off, obtained by integrating (2.3), (2.4), (2.6) numerically. Surface tension and the external pressure $p_{0}$ are chosen to vanish, and $L=4$. The initial conditions are $h(z, 0)=0.7-0.3 \cos (\pi z / L)$ and $C^{\prime}(z, 0)=0.2 \cos (\pi z / L)$. A positive source strength corresponds to a radial velocity field pointing inward, provoking pinch-off.

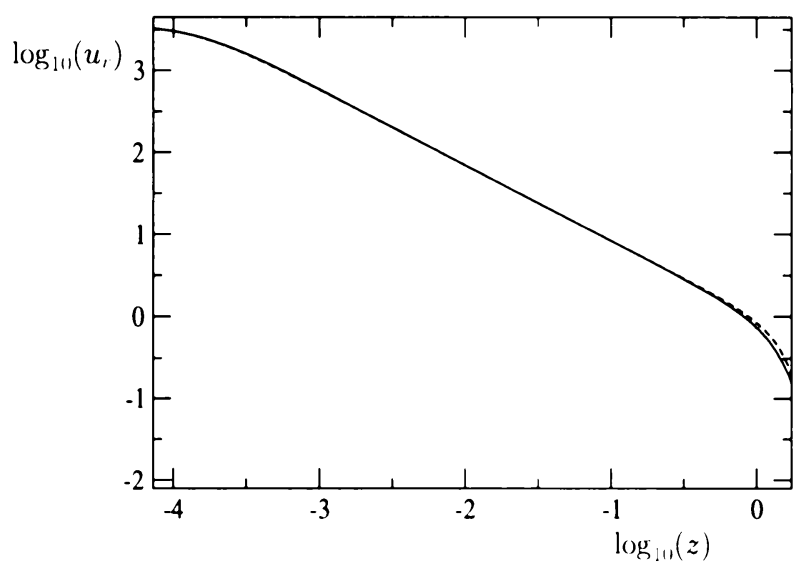

FIG. 2.2. Comparison of the radial velocity field as given by (2.4) (solid line) with the local approximation (2.13) (dashed line) for $\left|t^{\prime}\right|=10^{-5}$.

the experiment was very similar to that of Figure 1.1. A bubble of sulfur hexafluoride was released from an underwater nozzle of radius $R_{0}=0.235 \mathrm{~cm}[3]$. A sequence of four profiles, with a time distance of $1.5 \times 10^{-4}$ s between them, is shown in Figure 2.3 as the solid lines. This is compared to the simulation, at corresponding values of the dimensionless radius $h_{\min } / R_{0}$ (dashed lines). This does not yet fix the axial length scale, which was adjusted in the simulation by multiplying the $z$-axis by a constant factor. However, this was done only once, using the profile closest to pinch-off; the same factor was used for all the other profiles. The comparison is very convincing, emphasizing once more the practical validity of our approximations: use of the leading order expansion in the slenderness, as well as neglect of gravity and surface tension. Each of these approximations is, of course, also justified in an asymptotic sense as 


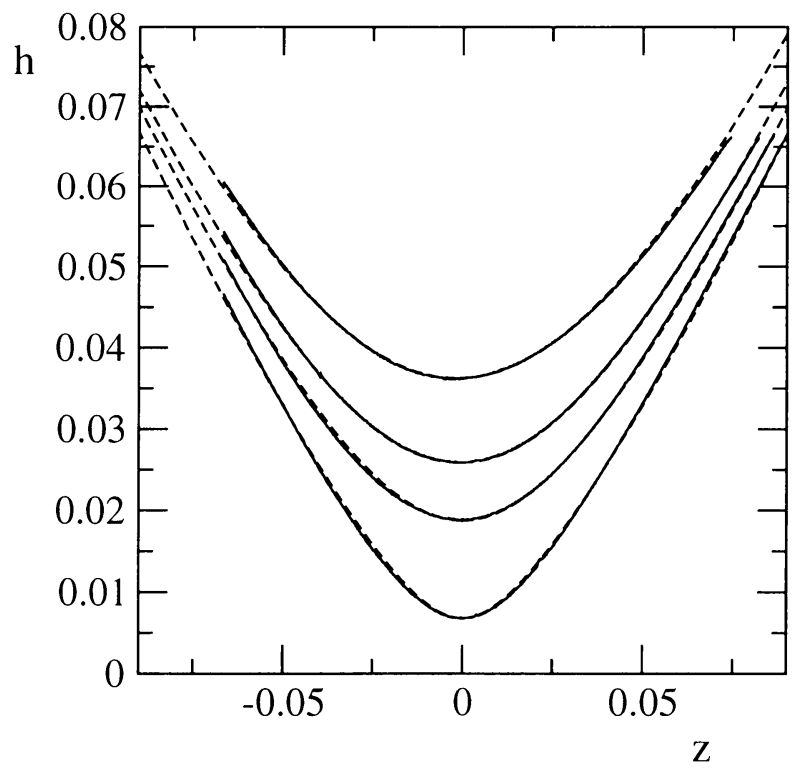

FIG. 2.3. A comparison between a sequence of experimental profiles of a bubble of sulfur hexafluoride pinching off in water [3]. The lines are the measured bubble radii in $\mathrm{cm}$ at a rate of 6688 frames per second; the dashed lines come from a simulation of (2.16). The initial conditions are $a(z, 0)=0.7+0.3(z / L)^{4}$ and $\dot{a}(z, 0)=0.1\left((z / L)^{2}-1\right)$. The four theoretical profiles have been timed to give the correct minimum radius; the axial scale of the profile has been adjusted once, using the last profile; and the other profiles use the same axial scale.

pinch-off is approached. Note as well that the experimental profile is extremely close to being symmetric about the pinch-point, even though the experiment is not up-down symmetric. In section 4 below we will calculate the asymptotic shape of the profile explicitly, thus confirming that it is indeed symmetric. All comparisons to numerical data to be reported below, used to check the validity of our asymptotic analysis, will be based on the simulation shown in Figure 2.3.

2.4. Strategy. In the following sections, we will analyze (2.16) using asymptotics. The idea is to write the solution in the form

$$
a(z, t)=a_{0}\left(t^{\prime}\right) A(\eta, \tau), \quad \eta=z / \Delta\left(t^{\prime}\right) .
$$

Thus the main time dependence is in the scale factors $a_{0}\left(t^{\prime}\right)$ and $\Delta\left(t^{\prime}\right)$, while the subleading time dependence is expected to be captured by the dependence of $A$ on $\tau=-\ln t^{\prime}$. The corresponding similarity form for $\ddot{a}(z, t)$ is

$$
\ddot{a}(z, t)=\ddot{a}_{0}\left(t^{\prime}\right) \phi(\eta, \tau) .
$$

In previous examples of drop pinch-off [10], the scales $a_{0}, \Delta$ were simply power laws. In the present case, these scales acquire logarithmic corrections. As a consequence the aspect ratio $\epsilon$ will turn out to vary as

$$
\epsilon \equiv \frac{a_{0}^{1 / 2}}{\Delta} \sim e^{-\frac{1}{2} \sqrt{\tau}}
$$

which goes to zero as $\tau \rightarrow \infty$. Indeed, the neck becomes increasingly slender upon approaching the pinch-point, ensuring that the analysis is self-consistent. 
The similarity functions and their time dependences will be computed using the following steps:

(a) First, in section 3 , we focus on the time dependence of $a_{0}, \Delta$. This time dependence can be found from expanding $a(z, t)$ and $\ddot{a}(z, t)$ around the minimum at $z=0$, yielding a dynamical equation for $a_{0}(t)$ and $\Delta(t)$. Solving this system, we find that $a_{0}=C_{0} t^{\prime} e^{-\sqrt{\tau}}$ and $\Delta^{2}=\sqrt{e} t^{\prime} / 4$ (see (3.12) below).

(b) In the following section 4 we derive a simplified local expression for the integral on the left of (2.16), using the time dependence of the scale factors found in (a). The result is (4.8).

(c) We then determine the similarity profiles $A(\eta, \tau)$ and $\phi(\eta, \tau)$ as they appear in (2.17) and (2.18). This is first done based on the local approximation (section 5), by taking the distinguished limit of keeping $\eta$ fixed as $\tau \rightarrow \infty$. This provides the "inner" part of the neck profile. The acceleration $\ddot{a}$ converges to a Lorentz peak at fixed $\eta$.

(d) Finally, in section 6 we find the "outer" profile of $\ddot{a}$, away from the central region where it has a sharp peak. This asymptotics applies to the case where $\eta e^{-\sqrt{\tau} / 2} \gg 1$.

3. The time dependence. Our aim is to explain the observed scaling behavior of the minimum cross section $a_{0} \equiv a_{0}(t) \equiv a(0, t)$, as well as of the axial length scale $\Delta$ of the profile, which can be characterized by the inverse curvature $\Delta \equiv\left(2 a_{0} / a_{0}^{\prime \prime}\right)^{1 / 2}$; cf. (2.7). By definition, we write $a_{0}^{\prime \prime} \equiv a_{0}^{\prime \prime}(t) \equiv\left(\partial_{z}^{2} a\right)(0, t)$ for the curvature of the profile $a(z, t)$ at the pinch-point. As shown later, and confirmed numerically in Figure 3.1, to leading order $\ddot{a}(z, t)$ behaves as

$$
\ddot{a}(z, t)=\frac{\ddot{a}_{0}(t)}{1+(z / \Delta)^{2}} .
$$

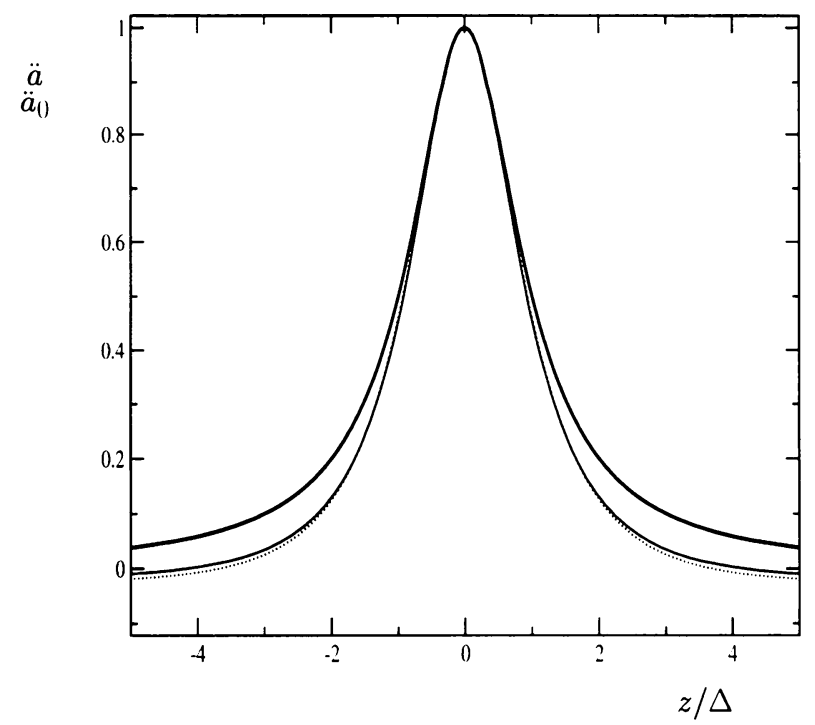

FIG. 3.1. Convergence of the central peak of ä toward (3.1). The heavy line is the Lorentz curve $1 /\left(1+\eta^{2}\right)$. The solid $\left(\left|t^{\prime}\right|=10^{-6}\right)$ and dotted $\left(\left|t^{\prime}\right|=10^{-3}\right)$ lines are the result of a numerical simulation of (2.16), rescaled according to (3.1). The initial conditions are the same as in Figure 2.1. Note that the acceleration becomes negative in the tails of the peak; this will be explained in section 6 (cf. (6.6)). 
But this means that, by evaluating (2.15) and its second derivative at $z=0$, we obtain a closed system of equations for $a_{0}$ and $\Delta$. The quadratic decay of (3.1) ensures that contributions to the integral are local, as anticipated above.

Using (3.1), we can evaluate the integral at $z=0$ explicitly:

$$
\int_{-\infty}^{\infty} \frac{\ddot{a}_{0}(t) d \xi}{\left(1+(\xi / \Delta)^{2}\right) \sqrt{\xi^{2}+a_{0}}}=\frac{\ddot{a}_{0}(t)}{\sqrt{1-a_{0} / \Delta^{2}}} \ln \left(\frac{1+\sqrt{1-a_{0} / \Delta^{2}}}{1-\sqrt{1-a_{0} / \Delta^{2}}}\right) .
$$

We rewrite the aspect ratio $a_{0} / \Delta^{2}=a_{0}^{\prime \prime} / 2$, so that for $a_{0}^{\prime \prime} \ll 1$ and $z=0,(2.16)$ simplifies to

$$
\ddot{a}_{0} \ln \left(\frac{8}{a_{0}^{\prime \prime}}\right)=\frac{\dot{a}_{0}^{2}}{2 a_{0}}
$$

Next, taking the second derivative of $(2.16)$, we obtain from a similar calculation

$$
\ddot{a}_{0}^{\prime \prime} \ln \left(\frac{8}{e^{3} a_{0}^{\prime \prime}}\right)-2 \frac{\ddot{a}_{0} a_{0}^{\prime \prime}}{a_{0}}=\frac{\dot{a}_{0} \dot{a}_{0}^{\prime \prime}}{a_{0}}-\frac{\dot{a}_{0}^{2} a_{0}^{\prime \prime}}{2 a_{0}^{2}} .
$$

As seen from a calculation analogous to that of the appendix, (3.3) and (3.4) are accurate up to corrections of order of the square of the aspect ratio, and thus consistent with our earlier approximations.

Following [12], we now rewrite (3.3), (3.4) as equations for the local (timedependent) exponents

$$
2 \alpha \equiv-\partial_{\tau} a_{0} / a_{0}, \quad 2 \delta \equiv-\partial_{\tau} a_{0}^{\prime \prime} / a_{0}^{\prime \prime} .
$$

Note that (3.5) is equivalent to taking the slope of a log-log plot but differs from $h_{0} \propto t^{\prime \alpha}$ if $\alpha$ is time-dependent. The result is

$$
\begin{aligned}
& \left(\alpha_{\tau}+\alpha-2 \alpha^{2}\right) \ln \left(\frac{8}{a_{0}^{\prime \prime}}\right)=-\alpha^{2} \\
& \left(\delta_{\tau}+\delta-2 \delta^{2}\right) \ln \left(\frac{8}{e^{3} a_{0}^{\prime \prime}}\right)=2 \alpha-3 \alpha^{2}-2 \alpha \delta+2 \alpha_{\tau},
\end{aligned}
$$

where the subscript denotes the $\tau$-derivative. The time dependence of $a_{0}^{\prime \prime}$ is found from integrating

$$
\ln \left(a_{0}^{\prime \prime}\right)_{\tau}=-2 \delta
$$

Compared to our earlier work [12], in (3.6) and (3.7) we have now computed all constants inside the logarithms. This is possible using the explicit shape of the acceleration profile (3.1), to be derived below; we conclude that $\Gamma_{1}=8$ and $\Gamma_{2}=8 / e^{3}$ in the notation of [12].

The behavior of the third order system (3.6)-(3.8) in the limit $\tau \rightarrow \infty$ is determined by the neighborhood of the fixed point $(\alpha, \delta, v)=(1 / 2,0,0)$. Putting $\alpha(\tau)=1 / 2+u(\tau)$ and $v(\tau)=1 / \ln \left(a_{0}^{\prime \prime}\right)$, the leading order behavior of (3.6)-(3.8) becomes

$$
u_{\tau}=u+v / 4, \quad \delta_{\tau}=-\delta-v / 4, \quad v_{\tau}=2 \delta v^{2} .
$$

The linearization around the fixed point thus has the eigenvalues 1,0 , and -1 . As explained, for instance, by [13] and [24], the positive eigenvalue corresponds to a 


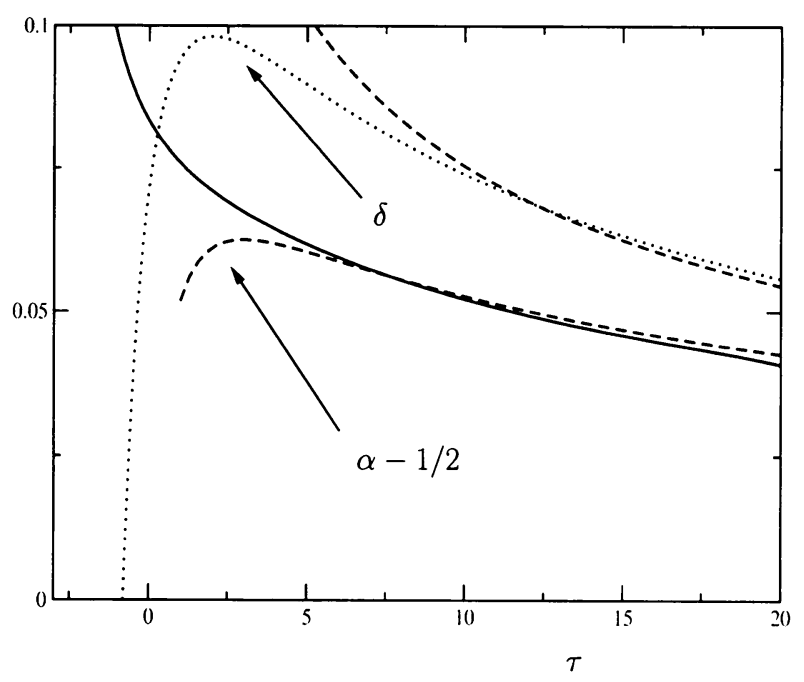

FIG. 3.2. Approach of the exponents to their asymptotic value, obtained from a numerical simulation of (2.16). The initial conditions are the same as in Figure 2.1. The solid line is $\alpha-1 / 2$; the dotted line is $\delta$. The two dashed lines are the predictions (3.11), but with a shift in the value of $\tau$, which is the same for the two curves.

change of the unknown origin of time and has no dynamical significance. On the other hand, the vanishing eigenvalue is the origin of the slow approach to the fixed point observed for the present problem. The derivatives $u_{\tau}$ and $\delta_{\tau}$ are of lower order in the first two equations of (3.9), and thus to leading order $u=\delta$ and $v=-4 \delta$. This means that the last equation of (3.9) can be simplified to

$$
\delta_{\tau}=-8 \delta^{3}
$$

The high (third order) nonlinearity explains the extremely slow approach to the scaling limit.

The approach to fixed-point behavior is best found by expanding (3.6)-(3.8) in powers of $\tau^{-1 / 2}$, fractional powers providing the right balance:

$$
\alpha=\frac{1}{2}+\frac{1}{4 \sqrt{\tau}}-\frac{1}{4 \tau}+O\left(\tau^{-3 / 2}\right), \quad \delta=\frac{1}{4 \sqrt{\tau}}+O\left(\tau^{-3 / 2}\right) .
$$

Knowing the constants $\Gamma_{1}, \Gamma_{2}$ (cf. equations (4) and (5) in [12]), we were able to calculate the exponents to order $1 / \tau$, going beyond the previously known result. In particular, (3.11) implies that the coefficients are universal at this order.

We also explored full numerical solutions to (3.6)-(3.8). Owing to the positive eigenvalue, initial conditions must be chosen from a two-dimensional subspace to reach the fixed point. However, in spite of having an additional adjustable parameter, the numerical solution did not significantly expand the range over which exponents could be predicted. Therefore, in Figure 3.2 we show only the comparison between the expansion (3.11) and the exponent values obtained from a numerical simulation of (2.16). The time dependence of $\alpha$ and $\delta$ differs only at order $1 / \tau$, so it is only with the new result (3.11) at hand that we can perform a meaningful comparison of both $\alpha$ and $\delta$. Equation (2.16) is invariant under a rescaling of time, i.e., a shift in $\tau$. Such a shift in $\tau$ is therefore determined by the initial conditions and has to be adjusted in 
a comparison of the exponents, as we did in Figure 3.2; of course, the shift was the same for both curves.

Our theory was validated further by [14], who simulated bubble breakup in a variety of systems, such as the wake of an impacting disk and gas bubbles detaching from an orifice. The value of $\alpha$ was plotted as a function of $a_{0}^{\prime \prime}$, which for all cases displayed the universal behavior of (3.6). To achieve a comparison without adjustment of parameters, [14] relied on the calculation of the constant $\Gamma_{1}$, first performed in the present paper. Interestingly, the time scales over which these quantities evolve were found to differ by orders of magnitude, depending on the physical realization of the collapse. This once more reflects the invariance under a rescaling of time of the asymptotic regime.

Note that (3.11) is also consistent with previously published experiments, where exponents were determined from fitting a straight line to a doubly logarithmic plot over a limited range. As a result of the limited experimental time resolution, values of alpha slightly larger than $1 / 2$ were reported. For example, [23] found $\alpha=0.57 \pm 0.03$, where smaller values of $\alpha$ were reported if the time resolution was increased for the same experiment (cf. Figures 5 and 10 in [23]). The width of the profile was also investigated in detail, and its scaling exponent was consistently found to be very close to $1 / 2$, in agreement with $(3.13)$ below.

The expansion (3.11) yields estimates for the leading order time dependencies of various key quantities:

$$
\begin{gathered}
a_{0}=C_{0} t^{\prime} e^{-\sqrt{\tau}}, \quad \dot{a}_{0}=-C_{0} e^{-\sqrt{\tau}}\left(1+\frac{1}{2 \sqrt{\tau}}\right), \\
\ddot{a}_{0}=\frac{C_{0}}{2 t^{\prime} \sqrt{\tau}} e^{-\sqrt{\tau}}\left(1+\frac{1}{2 \sqrt{\tau}}+O\left(\tau^{-1}\right)\right),
\end{gathered}
$$

where $C_{0}$ is a prefactor which depends on initial conditions. Inserting this back into (3.3), one finds the approximation

$$
a_{0}^{\prime \prime} \approx \frac{8}{\sqrt{e}} e^{-\sqrt{\tau}}, \text { and thus } \Delta=\left(\frac{\sqrt{e}}{4} t^{\prime}\right)^{1 / 2} .
$$

We conclude that the aspect ratio $\epsilon \equiv h_{0} / \Delta$ indeed behaves as anticipated in (2.19), so our calculation is self-consistent. In particular, neglected terms are exponentially small (in terms of $\tau$ ) compared to terms in the series expansion (3.11).

4. Local approximation. Now we compute the spatial structure of the pinch region, that is, the similarity function $A(\eta, \tau)$ in $(2.17)$. This will also yield the corresponding profile of $\ddot{a}$, which is a Lorentzian according to (3.1). To this end we derive a localized version of the integral equation (2.16), which captures the leading order asymptotics of the problem. The key is to recognize that significant accelerations $\ddot{a}(z, t)$, which determine the region over which there are significant contributions to the integral in (2.16), occur only over the scale $\Delta$. We localize the integral by isolating this region and splitting the integral according to

$$
\int_{-\infty}^{\infty} \frac{\ddot{a}(\xi, t) d \xi}{\sqrt{(z-\xi)^{2}+a(z, t)}}=\int_{-\Delta+z}^{\Delta+z} \ldots d \xi+\int_{\Delta+z}^{\infty} \ldots d \xi+\int_{-\infty}^{-\Delta+z} \ldots d \xi
$$

Now we consider the integrals on the right-hand side of (4.1) one by one. 
The first integral on the right-hand side of (4.1) can be rewritten as

$$
\int_{-\frac{\Delta}{\sqrt{u(z . t)}}}^{\frac{\Delta}{\sqrt{u(z .1)}}} \frac{\ddot{a}(z+\bar{\xi} \sqrt{a(z, t)}, t) d \bar{\xi}}{\sqrt{\bar{\xi}^{2}+1}} .
$$

If $z$ is of order $\Delta$, it follows from (3.1) that the limits of (4.2) can be approximated as

$$
\Delta / \sqrt{a(z, t)} \approx \Delta / h_{0} \equiv \epsilon^{-1} \propto e^{\sqrt{\tau} / 2},
$$

where we have used (2.19). This is large in the limit $\tau \rightarrow \infty$ that we are interested in. Now we expand the numerator of the integrand in (4.2) according to

$$
\ddot{a}(z+\bar{\xi} \sqrt{a(z, t)}, t)=\ddot{a}(z, t)+\ddot{a}^{\prime \prime}(z, t) a(z, t) \frac{\bar{\xi}^{2}}{2}+O\left(\bar{\xi}^{4}\right) .
$$

Using the integral

$$
\int_{-\epsilon^{-1}}^{\epsilon^{-1}} \frac{d \xi}{\sqrt{\xi^{2}+1}}=2 \ln \left(\epsilon^{-1}+\sqrt{\epsilon^{-2}+1}\right)
$$

the contribution of the first term of $(4.3)$ to $(4.2)$ is

$$
\ddot{a}(z, t) \ln \left(\frac{4 \Delta^{2}}{a(z, t)}\right) \approx \ddot{a}_{0} \ln \left(\epsilon^{-2}\right) \times \frac{1}{e^{\tau} t^{\prime}} .
$$

The second term of (4.3), using

$$
\int_{-\epsilon^{-1}}^{\epsilon^{-1}} \frac{d \xi \xi^{2}}{\sqrt{\xi^{2}+1}}=\epsilon^{-1}+\sqrt{\epsilon^{-2}+1}-\ln \left(\epsilon^{-1}+\sqrt{\epsilon^{-2}+1}\right),
$$

leads to

$$
\frac{\ddot{a}^{\prime \prime}(z, t) \Delta^{2}}{2} \approx \frac{\ddot{a}_{0}^{\prime \prime} \Delta^{2}}{2} \approx-\frac{1}{2 e^{\tau} \sqrt{\tau} t^{\prime}}
$$

Clearly, (4.5) is small compared to (4.4) in the limit $\tau \rightarrow \infty$, and so only the first term of the expansion (4.3) needs to be considered. The fourth order correction is even smaller.

Next we evaluate the second integral on the right-hand side of (4.1). Using the form (3.1) of $\ddot{a}(z, t)$, the integral can be estimated as

$$
\int_{\Delta+z}^{\infty} \frac{\ddot{a}(\xi, t) d \xi}{\sqrt{(z-\xi)^{2}+a(z, t)}} d \xi \approx \int_{\Delta+z}^{\infty} \frac{\ddot{a}_{0} \Delta^{2}}{\xi^{3}} d \xi=\frac{\ddot{a}_{0} \Delta^{2}}{2(\Delta+z)^{2}} \approx \frac{\ddot{a}_{0}}{2}
$$

if $z$ is of order $\Delta$. Clearly, (4.6) is small compared to the leading contribution (4.4) as $\epsilon \rightarrow 0$. An identical argument holds for the third integral on the right-hand side of (4.1). In summary, we have found that (4.4) is the leading contribution to (4.1), and so we have

$$
\int_{-\infty}^{\infty} \frac{\ddot{a}(\xi, t) d \xi}{\sqrt{(z-\xi)^{2}+a(z, t)}} \approx-\ddot{a}(z, t) \ln \epsilon^{2}
$$




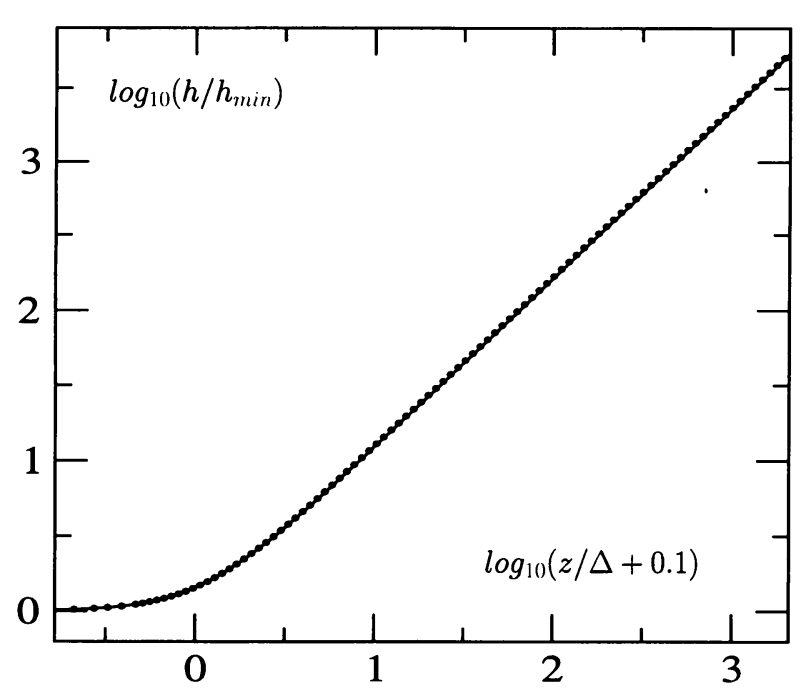

FIG. 4.1. Comparison between the local description (4.8) (small circles) and the integral equation (2.16) (solid line). The initial conditions are the same as in Figure 2.1. The scale is logarithmic, and a snapshot is taken for $\left|t^{\prime}\right|^{-6}$.

But this means that the localized version of (2.16) becomes

$$
-\ddot{a}(z, t) \ln \epsilon^{2}(\tau)=\frac{\dot{a}^{2}(z, t)}{2 a(z, t)},
$$

which is the formulation that is the basis for the calculations of the next section.

The description (4.8) is superficially similar to the scalar equation for the minimum radius given in [21] or [2] but differs in two important aspects. First, the $\operatorname{logarithm}$ of the aspect ratio $\ln \epsilon$ is replaced by the $\operatorname{logarithm}$ of the minimum radius itself; this yields an incorrect time dependence for the exponent $\alpha$; cf. (3.11). Second, (4.8) is an equation for the entire profile $a(z, t)$, not just for the minimum. As seen in Figure 4.1, the description is extremely good over a wide span in the rescaled variable, provided one is sufficiently close to pinch-off.

5. Neck profile: Inner region. The spatial variable $z$ is only a parameter in (4.8), which thus can easily be solved. Namely, transforming the independent variable according to $\partial_{t}=\partial_{\tau} / t^{\prime}$, and the dependent variable according to $d=a / a_{\tau}$, we find the linear equation

$$
d_{\tau}-d=1-\frac{1}{\ln \epsilon^{2}(\tau)}
$$

Now using $\ln \left(\epsilon^{2}(\tau)\right)=-\sqrt{\tau}+\ln \epsilon_{0}^{2}$ and expanding, we have

$$
d_{\tau}-d=\nu+O\left(\tau^{-3 / 2}\right)
$$

where for ease of notation we have put $\nu=1-1 /(2 \sqrt{\tau})$. Taking only leading order corrections in $\tau$ into account, (5.2) can be integrated to give

$$
d=-C_{1}(z) e^{\tau}-\nu+O\left(\tau^{-3 / 2}\right)
$$


One more integration then gives

$$
\ln a(z, t) \approx C_{2}(z)-\int_{\tau_{1},}^{\tau} \frac{d \tau^{\prime}}{C_{1}(z) e^{\tau^{\prime}}+\nu} .
$$

To compute the similarity form (2.17) of $a(z, t)$, we need to evaluate (5.4) at a constant value of the similarity variable $\eta=z / \Delta \propto z / \sqrt{t}^{\prime}$. Thus in the limit of $t^{\prime} \rightarrow 0$, we have to consider (5.4) for $z \rightarrow 0$. The constant of integration $C_{2}(z)$ describes the initial condition for the profile on a spatial scale of order unity; in the limit, its argument is replaced by $z=0$. The constant of integration $C_{1}(z)$, on the other hand, is rescaled by a time-dependent factor and encodes the form of the profile in similarity variables. We must have $C_{1}(0)=0$, because otherwise the integral in (5.4) would remain finite, and $a(0, t)$ would not go to zero at the singularity, as required. For $C_{1}$ vanishing at the origin, on the other hand, we find from (5.4)

$$
\ln a_{0} \approx C_{2}(0)-\int_{\tau_{0}}^{\tau} \frac{d \tau^{\prime}}{\nu} \approx C_{2}(0)-\int_{\tau^{\prime \prime}}^{\tau}\left(1+1 /\left(2 \sqrt{\tau^{\prime}}\right)\right) d \tau^{\prime}=-\tau-\sqrt{\tau}+\ln C_{0},
$$

where the notation for the arbitrary constant $C_{0}$ has been chosen in accordance with the notation of section 2.4. As expected, (5.5) agrees with the earlier calculation of the scale factors; cf. (3.12).

Now we determine the spatial form of the similarity profile. The expansion of $C_{1}$ around $z=0$ is $C_{1}=B z^{2}+O\left(z^{3}\right)$. Since this is evaluated at constant $\eta$, the third order term is smaller than the leading term by a factor of $\sqrt{t^{\prime}}$ and can be dropped. To evaluate the integral in the limit $C_{1} \rightarrow 0$, we integrate by parts, to produce an integral that is of lower order in $\tau$. Namely, setting $\Xi=\ln \left(C_{1}+\nu e^{-\tau}\right)$, we find that

$$
\frac{\partial}{\partial \tau} \Xi=-\frac{\nu+O\left(\tau^{-3 / 2}\right)}{C_{1} e^{\tau}+\nu}
$$

and thus

$$
\ln a(z, t) \approx \frac{\Xi}{\nu}+\int_{\left.\tau_{1}\right)}^{\tau} \frac{\Xi}{4 \tau^{3 / 2} \nu^{2}} d \tau^{\prime}+\text { const. }
$$

Remembering that $C_{1} e^{\tau} \approx B z^{2} / t^{\prime}=e B \eta^{2} / 4$, the first term in (5.7) becomes

$$
\frac{\Xi}{\nu} \approx \frac{-\tau+\ln \left(1+e B \eta^{2} / 4\right)}{\nu}
$$

The remaining integral in (5.7) can now be evaluated by expanding the integrand in powers of $C_{1}$ and using $\nu \approx 1$ to leading order. The result is

$$
\frac{\ln \left(C_{1}+e^{-\tau}\right)}{4 \tau^{3 / 2}}=\frac{1}{4 \sqrt{\tau}}+\frac{1}{4 \tau^{3 / 2}}\left[C_{1} e^{\tau}+O\left(C_{1} e^{\tau}\right)^{2}\right] .
$$

The integral over the first term gives $-\sqrt{\tau} / 2$, which contributes to the correct value of the scaling factor $a_{0}$. The integral over the exponential can be estimated as

$$
\int^{\tau} \frac{C_{1} e^{\tau}}{\tau^{3 / 2}} d \tau \approx-\frac{2 C_{1} e^{\tau}}{\sqrt{\tau}} \propto \frac{\eta^{2}}{\sqrt{\tau}}
$$

for $\tau \rightarrow \infty$. But this, at constant $\eta$, is clearly of lower order than the nonuniversal constants that contribute to (5.7). Thus in summary we obtain from (5.7)

$$
\nu \ln a(z, t) \approx-\tau+\ln \left(1+e B \eta^{2} / 4\right)-\sqrt{\tau} / 2,
$$




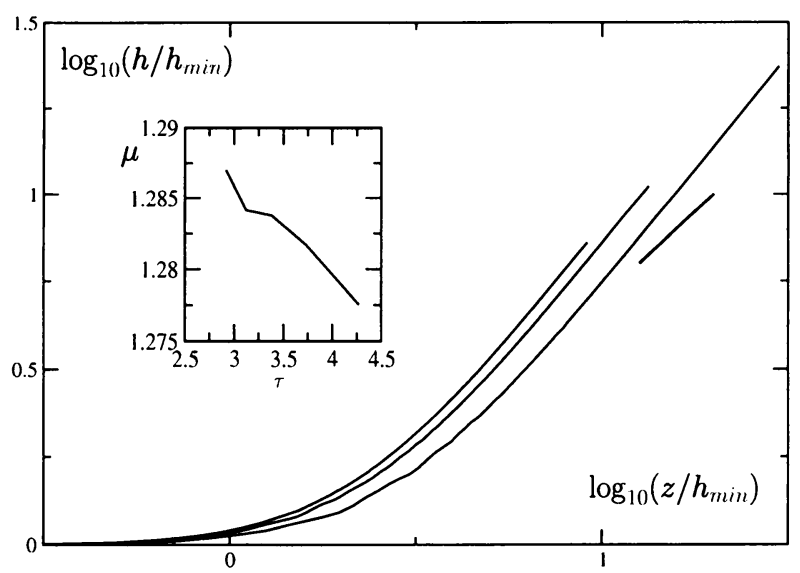

FIG. 5.1. The asymptotic behavior of a sequence of experimental profiles [23], 52, 27, and $7 \mu \mathrm{s}$ away from pinch-off, plotted on a logarithmic scale. The thick line shows a slope of one for comparison. The inset shows the slope of the profile, determined in the range $10^{0.6} \leq h / h_{\min } \leq 10$. The time scale used to nondimensionalize $t$ was chosen, somewhat arbitrarily, as $500 \mu s$.

and therefore

$$
a(z, t) \equiv a_{0} A(\eta, \tau) \approx a_{0}\left[1+\eta^{2}\right]^{1+\frac{1}{2 \sqrt{\tau}}} .
$$

Here we have made use of the fact that $\Delta^{2}=2 a_{0} / a_{0}^{\prime \prime}$, and thus $B=4 / e$.

In particular, this means that the profile $h(z, t)$ behaves like a power law with exponent $\mu \approx 1+1 /(2 \sqrt{\tau})$ for large values of $\eta$. The interesting and subtle feature here is that $\mu$ is varying logarithmically in time. We first attempted to confirm this prediction directly from experimental data; cf. Figure 5.1. It is seen clearly that $\mu$ is indeed greater than unity, and that it is decreasing in time. However, both the limited temporal and spatial resolution do not permit a fully quantitative comparison. We therefore determined the exponent of the profile (5.12) from the simulation described before, which follows $h_{0}$ through six orders of magnitude. The result (cf. Figure 5.2) shows good agreement with the predicted exponent, but only for large values of $\tau$; as usual, transients are very slow to decay. For typical values of $\tau$ as found experimentally, deviations between simulations and asymptotics are quite large.

The spatial dependence of $\dot{a}=a_{\tau} / t^{\prime} \equiv a /\left(v t^{\prime}\right)$ is easily found from (5.3):

$$
\dot{a}=\frac{a e^{-\tau}}{C_{1}(z) e^{\tau}-1} \approx \dot{a}_{0}\left(1+\eta^{2}\right)^{\frac{1}{\sqrt{2 \tau}}} .
$$

Now, with $a$ and $\dot{a}$ in hand, (4.8) can be used to find the similarity form of $\ddot{a}$, as defined by $(2.18)$ :

$$
\ddot{a} \equiv \ddot{a}_{0} \phi(\eta, \tau)=\ddot{a}_{0}\left(1+\eta^{2}\right)^{-1+\frac{1}{2 \sqrt{\tau}}} .
$$

In the limit of large $\tau$, this reduces to (3.1), as required, and as confirmed in Figure 3.1. Convergence in the tails of the peak, however, is slow, and the acceleration actually becomes negative. This is because the spatial integral over the acceleration must vanish, as we show now. 


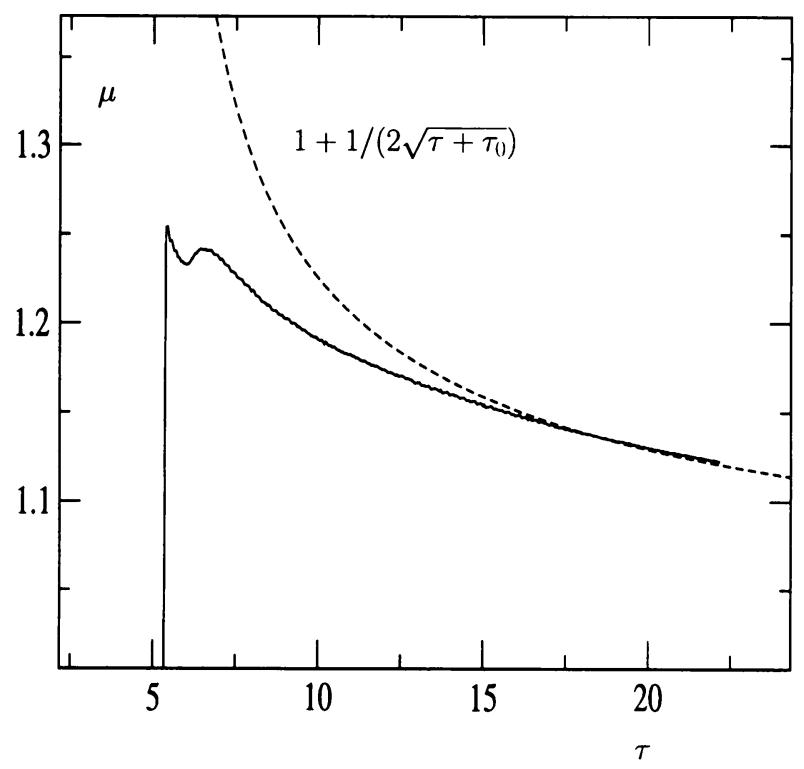

FIG. 5.2. The asymptotic slope $\mu$ of the profile, determined from a doubly logarithmic plot. We used a numerical simulation of (2.16), with initial conditions as in Figure 2.1. A linear regression was performed in the range $10 \leq h / h_{\min } \leq 100$. For large $\tau, \mu$ agrees well with the theoretical prediction $\mu \approx 1+1 /(2 \sqrt{\tau})$, allowing for a shift in time as usual.

6. Neck profile: Outer region. The above arguments, based on the local equation (4.8), predict the spatial structure of the profile in the inner region. By this we mean that the limit $\tau \rightarrow \infty$ is taken, keeping the similarity variable $\eta$ fixed. However, we will now show that this is not sufficient to get a global picture of the universal behavior near pinch-off. Instead, one has to introduce an outer region of the neck profile, where we describe the profile at a fixed size in the spatial variable $z$. For this, it is necessary to go back to the original equation (2.16), and to invert the integral kernel. Thus, writing (2.16) in similarity variables as in (2.17), (2.18), we obtain, using the result of the previous section,

$$
\int_{-\infty}^{\infty} \frac{\phi(\zeta, \tau) d \zeta}{\sqrt{(\eta-\zeta)^{2}+a\left(\eta t^{\prime 1 / 2}\right) / t^{\prime}}}=\frac{\sqrt{\tau}}{\left(1+B \eta^{2}\right)^{1-\frac{1}{\sqrt{\tau}}}}
$$

The right-hand side of (6.1) is valid for any finite $\eta$. But this means that $\eta t^{\prime 1 / 2}$ goes to zero in the limit $\tau \rightarrow \infty$, so can replace $a\left(\eta t^{\prime}\right) / t^{\prime} \approx a_{0} / t^{\prime} \approx e^{-\sqrt{\tau}}$ and solve $(6.1)$ by Fourier transform.

Using (A.4), we find

$$
\hat{\phi}(k)=\frac{\hat{s}(k) \sqrt{\tau}}{2 K_{0}\left(k e^{\sqrt{\tau} / 2}\right)},
$$

where

$$
\hat{s}(k)=\int_{-\infty}^{\infty} \frac{\cos (k \eta)}{\left(1+B \eta^{2}\right)^{1-\frac{1}{\sqrt{\tau}}}} d \eta \text { and } \hat{\phi}(k)=\int_{-\infty}^{\infty} \phi(\eta) \cos (k \eta) d \eta
$$




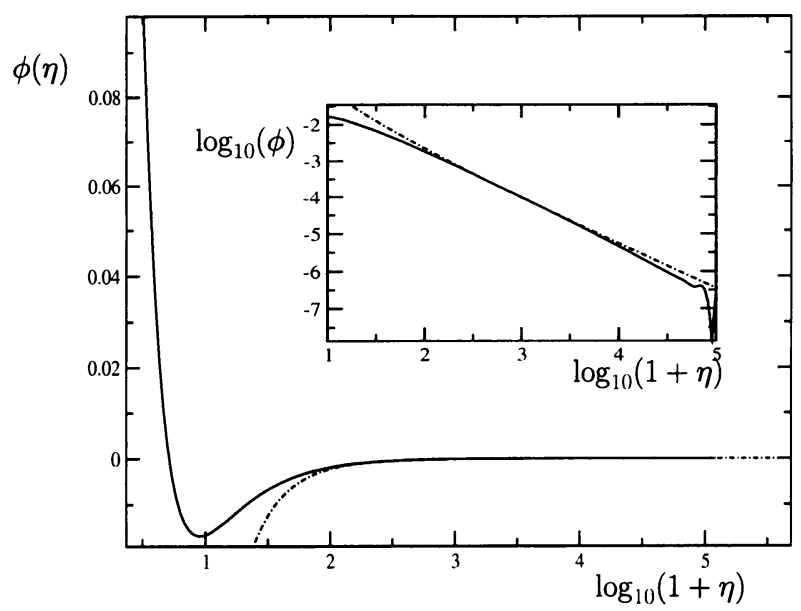

FIG. 6.1. The scaling function $\phi(\eta)$ corresponding to $\ddot{a}$, defined by $(2.18)$, is shown on a logarithmic scale. The profile was computed for $t^{\prime}=10^{-6}$. We used a numerical simulation of (2.16), with initial conditions as in Figure 2.1. In the center, there is a Lorentzian peak, but $\phi$ becomes negative for large arguments. In the inset, the decay of $\phi$ is compared to (6.9), which is shown as the dot-dashed line. The argument $1+\eta$ inside the logarithm was chosen simply for convenience, to be able to show part of the central region as well as the tail.

The inverse Fourier transform then gives the real space profile

$$
\phi(\eta)=\frac{\sqrt{\tau}}{4 \pi} \int_{-\infty}^{\infty} \frac{\hat{s}(k) \cos (k \eta)}{K_{0}\left(k e^{\sqrt{\tau} / 2}\right)} d k
$$

In the limit $\tau \rightarrow \infty, \hat{s}(k)$ becomes

$$
\hat{s}(k)=\frac{\pi}{\sqrt{B}} e^{-\frac{k}{\sqrt{13}}},
$$

and even the general case can be done in terms of Bessel functions. Since we want to compute $\phi(\eta)$ for large arguments, we are interested in the limit of small $k$ in which $\hat{s}$ becomes a constant and $K_{0}\left(k e^{\sqrt{\tau} / 2}\right) \approx-\ln \left(k e^{\sqrt{\tau} / 2}\right)=\sqrt{\tau} / 2-\ln k$. For $k \ll e^{-\sqrt{\tau} / 2}$, the constant can be neglected. Thus the first observation we can make is that $\hat{\phi}(0)=0$, so it follows from (6.3), putting $k=0$, that

$$
\int_{-\infty}^{\infty} \phi(\eta, \tau) d \eta=0 \text { and thus } \int_{-\infty}^{\infty} \ddot{a}(z, t) d z=0 .
$$

Clearly, our initial approximation (5.14), which predicts a positive acceleration, cannot apply uniformly: for large arguments $\phi$ must be negative, to ensure a vanishing total area under the graph of $\phi$. This is confirmed in Figure 6.1, where $\phi$ is seen to be positive in the center but negative for large arguments. To find the behavior in the outer region, we consider the case $\eta e^{-\sqrt{\tau} / 2} \gg 1$, for which (6.4) becomes

$$
\phi(\eta) \approx-\frac{\sqrt{\tau}}{4 \sqrt{B}} \int_{-\infty}^{\infty} \frac{\cos (k \eta) d k}{\ln k}
$$

With a change of variables, and integrating by parts, we find

$$
\int_{-\infty}^{\infty} \frac{\cos (k \eta) d k}{\ln k}=\frac{1}{\eta} \int_{-\infty}^{\infty} \frac{\sin y d y}{y(\ln (y / \eta))^{2}} \approx \frac{\pi}{\eta(\ln \eta)^{2}}
$$


In the last step we have neglected the slow $y$-dependence inside the logarithm, allowing us to perform the integral. This means we finally have

$$
\phi(\eta) \approx-\frac{\pi \sqrt{\tau}}{4 \eta \sqrt{B}(\ln \eta)^{2}}, \quad \eta e^{-\sqrt{\tau} / 2} \gg 1,
$$

so $\phi$ is negative as expected, to be consistent with (6.6). The asymptotic behavior (6.9) is compared to a numerical simulation in Figure 6.1, showing good agreement.

Thus to summarize, when the profile is considered at constant $\eta$, the result is (5.14). This approximation holds for $\eta \ll e^{\sqrt{\tau} / 2}$ and can be considered as the inner part of the similarity profile. In the opposite limit of $\eta \gg e^{\sqrt{\tau} / 2}$, one instead finds the outer profile (6.9). According to (6.6), the area of both parts adds up to zero.

7. Discussion. We have analyzed the collapse of an axisymmetric cavity in an inviscid fluid. It was found that the minimum neck radius, $h_{0}$, has a universal dynamics that is intimately coupled to the axial length scale, $\Delta$. This dynamics can be expressed in terms of an effective exponent that has a very slow logarithmic evolution, according to (3.11). We were able to calculate the exponents to higher order than in our previous paper [12]. For example, this permits distinguishing between the time dependence of the two exponents $\alpha$ and $\delta$.

However, the main results of this paper relate to the spatial structure of the profile, which were previously unknown. As seen from (5.12), the "near" tail of the profile is again characterized by an exponent which depends logarithmically on time. However, (5.12) does not describe the profile in a finite region of space as $t \rightarrow t_{0}$. This distinguishes the asymptotics of bubble pinch-off from other, superficially similar pinch-off problems, like the break-off of a fluid drop in air [9]. Instead, another asymptotic region needs to be investigated to find the "far" tail (6.9). Each asymptotic result has in this paper been checked quantitatively by comparison to numerical simulation.

We also went into great detail to test our numerical simulations, as well as our analytical results, directly against experiment. The agreement with numerical simulation is very good; this remains true if terms of leading order in the slenderness have been dropped. Our asymptotic results for scaling exponents agree with experiment as well, but their time dependence can be detected only as far as their trend is concerned, since one is restricted to a limited number of decades. Typical experimental values for the exponent $\alpha$ are about $0.56[4,16,18,23,2,8]$, although the precise value depends on initial conditions [2] and on the frame rate [23]. To interpret these findings in terms of our theory requires an estimate of $\tau \equiv-\ln t^{\prime}$, and we have at best an educated guess for the time scale in which to express $t^{\prime}$. However, at higher frame rates, but otherwise under the same experimental conditions, the exponent $\alpha$ decreases. The exponent of the width $\Delta$, on the other hand, remains very close to $1 / 2$ [23]. For the exponent $\mu$, characterizing the tail of the self-similar profile, we were able to detect a decreasing trend (cf. Figure 5.2), in agreement with theory.

Perhaps the best dimensionless parameter with which to parameterize the pinch process is the aspect ratio (2.8), for which $a_{0}^{\prime \prime} \sim \epsilon^{2}$. To leading order we found that $\sqrt{\tau} \simeq-\ln a_{0}^{\prime \prime}$, so that we can express the exponent as

$$
\alpha=\frac{1}{2}+\frac{1}{-4 \ln \left(a_{0}^{\prime \prime}\right)}+O\left(\frac{1}{\ln ^{2} a_{0}^{\prime \prime}}\right),
$$

as inferred readily from (3.6). This interpretation is consistent with experiments by [2], where cavities of different aspect ratios were created by forcing a disk through a 
water surface: higher impact velocities provide increasingly slender cavities. Indeed, the exponent was found to decrease logarithmically with the impact velocity, to values that approach $1 / 2$. We thus believe that such experiments explore different parts of a universal collapse that is governed by the square of the aspect ratio $a_{0}^{\prime \prime}$.

Appendix. Linear stability. It is instructive to perform a linear stability analysis around a cylindrical cavity, based on the equations of motion derived above. To maintain a stationary cavity of radius $h_{0}$, one needs a cavity pressure of $p_{0}=\gamma / h_{0}$ in (2.15). We set $h=h_{0}+\epsilon_{h}$, and thus $a=a_{0}+2 h_{0} \epsilon_{h}$, where $h_{0}$ is the unperturbed cavity radius. Since $C=O\left(\epsilon_{h}\right)$, we have to linear order

$$
\int_{-\infty}^{\infty} \frac{\dot{C} d \xi}{\sqrt{(z-\xi)^{2}+a_{0}}}=-\frac{\gamma}{\rho}\left(\frac{\epsilon_{h}}{h_{0}}+\epsilon_{h}^{\prime \prime}\right)
$$

where we have used

$$
\kappa=\frac{1}{h\left(1+h^{\prime 2}\right)^{1 / 2}}-\frac{h^{\prime \prime}}{\left(1+h^{\prime 2}\right)^{3 / 2}}
$$

for the mean curvature. The corresponding linearized version of (2.3) is

$$
-\frac{\dot{\epsilon_{h}}}{h_{0}}=\int_{-\infty}^{\infty} \frac{C d \xi}{{\sqrt{(z-\xi)^{2}+a_{0}}}^{3}} .
$$

Expanding the perturbation into plain waves, we set $\epsilon_{h}=e^{\omega t} \cos k z$. The integrals can now be done using using the Fourier transforms

(A.4)

$\int_{-\infty}^{\infty} \frac{\cos k \xi d \xi}{\sqrt{(z-\xi)^{2}+a_{0}}}=2 K_{0}\left(k h_{0}\right) \cos k z, \quad \int_{-\infty}^{\infty} \frac{\cos k \xi d \xi}{{\sqrt{(z-\xi)^{2}+a_{0}}}^{3}}=\frac{2 k}{h_{0}} K_{1}\left(k h_{0}\right) \cos k z$

where $K_{0}, K_{1}$ are modified Bessel functions of the second kind. Thus, combining (A.1) and (A.3), we find

$$
\omega^{2}=\omega_{0}^{2}\left(1-\left(k h_{0}\right)^{2}\right) \frac{k h_{0} K_{1}\left(k h_{0}\right)}{K_{0}\left(k h_{0}\right)}
$$

with the characteristic frequency $\omega_{0}=\sqrt{\gamma /\left(\rho h_{0}^{3}\right)}$. Reassuringly, this result is identical to the exact result as found in, e.g., [5]. If, on the other hand, the linearization is performed using the slender-body equation (2.15), the result is

$$
\omega^{2}=\omega_{0}^{2}\left(1-\left(k h_{0}\right)^{2}\right) \frac{1}{K_{0}\left(k h_{0}\right)} .
$$

Both (A.5) and (A.6) show that the instability is cut off at short wavelengths, owing to surface tension. Namely, for $k h_{0}>1$ the growth rate becomes complex.

Acknowledgments. We are very grateful to Justin Burton and Peter Taborek for making their unpublished data available to us, which is shown in Figure 2.3, and to Siggi Thoroddsen for providing us with the data shown in Figure 5.1. We also thank John Lister for useful remarks and criticism, and Stephan Gekle for pointing out an incorrect prefactor.

\section{REFERENCES}

[1] H. Ashley and M. Landahl, Aerodynamics of Wings and Bodies, Addison-Wesley, Reading, MA, 1965. 
[2] R. Bergmann, D. van der Meer. M. Stijnman. M. Sandtke. A. Prosperetti. and D. LoHSE, Giant bubble pinch-off, Phys. Rev. Lett.. 96 (2006), 154505.

[3] J. C. BURTON AND P. TABOREK, Bifurcation from bubble to droplet behavior in inviscid pinchoff, Phys. Rev. Lett., 101 (2008), 214502.

[4] J. C. Burton. R. WALDREP. AND P. TABOREK, Scaling and instabilities in bubble pinch-off, Phys. Rev. Lett., 94 (2005), 184502.

[5] S. Chandrasekhar, Hydrodynamic and Hydromagnetic Stability. Dover, New York, 1961.

[6] Y.-J. Chen And P. H. Steen, Dynamics of inviscid capillary breakup: Collapse and pinchoff of a film bridge, J. Fluid Mech., 341 (1997), pp. 245-267.

[7] R. F. DAY. E. J. Hinch. AND J. R. Lister, Self-similar capillary pinchoff of an inviscid fluid, Phys. Rev. Lett., 80 (1998), pp. 704-707.

[8] V. Duclaux. F. Caille. C. Duez. C. Ybert. L. Bocquet. and C. Clanet, Dynamics of transient cavities, J. Fluid Mech., 591 (2007), p. 1.

[9] J. EgGers, Universal pinching of 3D axisymmetric free-surface flow, Phys. Rev. Lett., 71 (1993), pp. 3458-3460.

[10] J. Eggers, Drop formation-An overview, ZAMM Z. Angew. Math. Mech., 85 (2005), pp. 400410.

[11] J. Eggers And T. F. Dupont, Drop formation in a one-dimensional approximation of the Navier-Stokes equation, J. Fluid Mech., 262 (1994). pp. 205-221.

[12] J. Eggers. M. A. Fontelos. D. Leppinen. And J. H. Snoeijer, Theory of the collapsing axisymmetric cavity, Phys. Rev. Lett., 98 (2007), 094502.

[13] S. Filippas And R. V. Kohn, Refined asymptotics for the blowup of $u_{t}-\delta u=u^{p}$. Comm. Pure Appl. Math., 45 (1992), pp. 821-869.

[14] S. Gekle, J. H. Snoeijer, D. Lohse. And D. VAn Der Meer, Approach to universality in axisymmetric bubble pinch-off, Phys. Rev. E. 80 (2009), 036305.

[15] J. M. Gordillo And M. A. Fontelos, Satellites in inviscid breakup of bubbles. Phys. Rev. Lett., 98 (2007), 144503.

[16] J. M. Gordillo, A. Sevilla. J. Rodriguez-Rodrigues. and C. Martinez-Bazan, Axisymmetric bubble pinch-off at high Reynolds numbers. Phys. Rev. Lett., 95 (2005), 194501.

[17] R. A. Handelsman And J. B. Keller, Axially symmetric potential flow around a slender body, J. Fluid Mech., 28 (1967), pp. 131-147.

[18] N. C. Keim. P. Møller. W. W. Zhang. And S. R. NAGel, Breakup of air bubbles in water: Memory and breakdown of cylindrical symmetry. Phys. Rev. Lett., 97 (2006), 144503.

[19] L. D. Landau and E. M. Lifshitz, Fluid Mechanics, Pergamon, Oxford, UK, 1984.

[20] M. S. Longuet-Higgins. B. R. Kerman. And K. Lunde, The release of air bubbles from an underwater nozzle, J. Fluid Mech., 230 (1991), p. 365.

[21] H. N. OG̃uz And A. Prosperetti, Dynamics of bubble growth and detachment from a needle, J. Fluid Mech., 257 (1993), p. 111.

[22] D. H. Peregrine, G. Shoker, and A. Symon, The bifurcation of liquid bridges, J. Fluid Mech., 212 (1990), pp. 25-39.

[23] S. T. Thorodden, E. G. Etoh, and K. Takeara, Experiments on bubble pinch-off, Phys. Fluids, 19 (2007), 042101.

[24] J. J. L. Velázquez. V. A. Galaktionov. and M. A. Herrero, The space structure near a blow-up point for semilinear heat equations: A formal approach. Comput. Math. Math. Phys., 31 (1991), pp. 46-55. 